\title{
FULLY EXPLICIT QUASICONVEXIFICATION OF THE MEAN-SQUARE DEVIATION OF THE GRADIENT OF THE STATE IN OPTIMAL DESIGN
}

\author{
PABLO PEDREGAL
}

(Communicated by Stuart Antman)

\begin{abstract}
We explicitly compute the quasiconvexification of the resulting integrand associated with the mean-square deviation of the gradient of the state with respect to a given target field, when the underlying optimal design problem in conductivity is reformulated as a purely variational problem. What is remarkable, more than the formula itself, is the fact that it can be shown to be the full quasiconvexification.
\end{abstract}

\section{INTRODUCTION}

In a number of recent papers ([1], [10]), a variational approach to optimal design and structural optimization has been proposed as an alternative to the analysis of some of these problems. In particular, we would like to focus in this note on one such typical situation in conductivity, where two different conducting materials, with conductivities $\alpha$ and $\beta, 0<\alpha<\beta$, are to be mixed, to fill out a simplyconnected design domain $\Omega \subset \mathbb{R}^{2}$, so as to minimize the integral of the square of the gradient of the electric potential $u$ over $\Omega$. In more precise terms, given $\Omega$, $u_{0} \in H^{1}(\Omega), f \in L^{2}(\Omega), \alpha, \beta$, as above, we want to

$$
\text { Minimize } I(\chi)=\int_{\Omega}|\nabla u(x)-f(x)|^{2} d x
$$

where $\chi$ is the characteristic function of a subset of $\Omega$ and $u \in H^{1}(\Omega)$ is the unique solution of

$$
\begin{aligned}
\operatorname{div}((\alpha \chi(x)+\beta(1-\chi(x))) \nabla u(x)) & =0 \quad \text { in } \Omega, \\
u & =u_{0} \quad \text { on } \partial \Omega .
\end{aligned}
$$

Such problems are known to lack optimal solutions within the class of characteristic functions since the pioneering work 8]. Lately, this particular problem has been studied (4], 7], 12]) by trying to extend the ideas of homogenization to tackle the dependence on derivatives of states. We will further comment on this in the last section. For a recent, very good introduction to homogenization and its application

Received by the editors March 15, 2001 and, in revised form, June 27, 2001

2000 Mathematics Subject Classification. Primary 49J45, 74P10.

I would like to acknowledge several stimulating conversations with R. Lipton concerning the type of optimal design problems considered here and to J. C. Bellido for carrying out various initial computations. I also appreciate the criticism of several referees which led to the improvement of several aspects of this note. 
to optimal design see [13. Our approach here is different and tries to avoid the nonlocal nature of the equilibrium law by introducing a suitable potential as a new independent field (see the references cited above). It is also interesting to point out that in the particular case $f \equiv 0$ the optimal solution of the problem corresponds to the choice $\chi \equiv 0$ or $\chi \equiv 1$ (either pure phase) since in this case we obtain the harmonic function with boundary values $u_{0}$. In a slightly different context the case $f \equiv 0$ has also been shown to have an optimal solution by Lipton (see [4]). For $f$ not identically zero, it is plausible that optimal solutions do not exist.

Under the hypothesis of simple connectedness of $\Omega$, there exists a potential $v \in$ $H^{1}(\Omega)$ such that

$$
\operatorname{div}((\alpha \chi(x)+\beta(1-\chi(x))) \nabla u(x))=0 \quad \text { in } \Omega
$$

is equivalent to

$$
(\alpha \chi(x)+\beta(1-\chi(x))) \nabla u(x)+T \nabla v(x)=0,
$$

where $T$ is the counterclockwise $\pi / 2$-rotation in the plane. If we collect both $u$ and $v$ in a single vector field $U=(u, v)$, it is not hard to realize that our initial optimal design problem is equivalent to

$$
\text { Minimize } I(U)=\int_{\Omega} W(x, \nabla U(x)) d x
$$

subject to

$$
U \in H^{1}(\Omega), \quad U^{(1)}=u_{0} \quad \text { on } \partial \Omega,
$$

where the density

$$
W: \mathbf{M}^{2 \times 2} \rightarrow \mathbb{R}^{*}=\mathbb{R} \cup\{+\infty\}
$$

is defined by

$$
W(x, A)= \begin{cases}\left|A^{(1)}-f(x)\right|^{2} & \text { if } A \in \Lambda_{\alpha} \cup \Lambda_{\beta}, \\ +\infty & \text { otherwise. }\end{cases}
$$

Here $U^{(i)}, i=1,2$, denotes the $i$-th component of $U, A^{(i)}, i=1,2$, denotes the $i$-th row of $A$, and $\Lambda_{\gamma}$ denotes the two-dimensional subspace of matrices defined by

$$
\Lambda_{\gamma}=\left\{A \in \mathbf{M}^{2 \times 2}: \gamma A^{(1)}+T A^{(2)}=0\right\} .
$$

Since it is equivalent to our original optimal design problem, this new vector variational problem does not admit optimal solutions. In such cases, however, its relaxation ([3]) usually provides all the information needed to understand and approximate the optimal behavior. This relaxation amounts to computing the quasiconvexification of $W$ and determining optimal Young measures ([9]). This is the main accomplishment of this work.

Theorem. Put

$$
\begin{aligned}
g(A)= & \alpha^{2} \beta^{2}\left|A^{(1)}\right|^{4}+\left|A^{(2)}\right|^{4}+\left(\alpha^{2}+6 \alpha \beta+\beta^{2}\right) \operatorname{det} A^{2} \\
& -2 \alpha \beta\left|A^{(1)}\right|^{2}\left|A^{(2)}\right|^{2}-2 \alpha \beta(\alpha+\beta)\left|A^{(1)}\right|^{2} \operatorname{det} A-2(\alpha+\beta)\left|A^{(2)}\right|^{2} \operatorname{det} A, \\
h(A)= & (\alpha+\beta) \operatorname{det} A-\alpha \beta\left|A^{(1)}\right|^{2}-\left|A^{(2)}\right|^{2},
\end{aligned}
$$


and consider the set of matrices

$$
\Gamma=\left\{A \in \mathbf{M}^{2 \times 2}: h(A) \geq 0, g(A) \geq 0\right\} .
$$

Then the quasiconvexification $Q W$ of $W$ is given by

$$
\begin{aligned}
Q W(x, A)= & \frac{1}{2 \alpha \beta}\left[\alpha \beta\left|A^{(1)}\right|^{2}-\left|A^{(2)}\right|^{2}+(\alpha+\beta) \operatorname{det} A-\sqrt{g(A)}\right] \\
& -2 A^{(1)} \cdot f(x)+|f(x)|^{2}
\end{aligned}
$$

if $A \in \Gamma$, and

$$
Q W(x, A)=+\infty
$$

otherwise. Moreover the unique optimal Young measure providing the value of this quasiconvexification, when $A$ does not belong to $\Lambda_{\alpha} \cup \Lambda_{\beta}$, is the first-order laminate

$$
t \delta_{A^{\alpha}}+(1-t) \delta_{A^{\beta}}
$$

where

$$
\begin{aligned}
& t=\frac{1}{2}+\frac{1}{2(\beta-\alpha) \operatorname{det} A}\left[\alpha \beta\left|A^{(1)}\right|^{2}-\left|A^{(2)}\right|^{2}+\sqrt{g(A)}\right], \\
& A^{\alpha}=\left(\begin{array}{c}
z \\
\alpha T z
\end{array}\right), \quad z=\frac{1}{t} \frac{1}{\beta-\alpha}\left(\beta A^{(1)}+T A^{(2)}\right) \\
& A^{\beta}=\left(\begin{array}{c}
w \\
\beta T w
\end{array}\right), \quad w=\frac{1}{(1-t)} \frac{1}{\alpha-\beta}\left(\alpha A^{(1)}+T A^{(2)}\right) .
\end{aligned}
$$

\section{Sketch OF PROOF}

We divide the proof in several steps.

Step 1. We know that in computing $Q W(x, A)$ we must care about (homogeneous) gradient Young measures supported in the set where $W$ is finite and with first moment $A([9)$. Therefore, let $\nu$ be such a gradient Young measure supported in $\Lambda_{\alpha} \cup \Lambda_{\beta}$ with first moment $A$. Naturally we can decompose $\nu$ into two parts

$$
\nu=s \nu_{\alpha}+(1-s) \nu_{\beta}, \quad s \in[0,1]
$$

where

$$
\operatorname{supp}\left(\nu_{\alpha}\right) \subset \Lambda_{\alpha}, \quad \operatorname{supp}\left(\nu_{\beta}\right) \subset \Lambda_{\beta} .
$$

In the same way, set

$$
A_{\alpha}=\int_{\Lambda_{\alpha}} F d \nu_{\alpha}(F) \in \Lambda_{\alpha}, \quad A_{\beta}=\int_{\Lambda_{\beta}} F d \nu_{\beta}(F) \in \Lambda_{\beta}
$$

It is also easy to check the following:

$$
\begin{gathered}
\operatorname{det} A=-A^{(1)} \cdot T A^{(2)}, \\
\operatorname{det} A=\alpha\left|A^{(1)}\right|^{2} \quad \text { if } A \in \Lambda_{\alpha}, \\
\operatorname{det} A=\beta\left|A^{(1)}\right|^{2} \quad \text { if } A \in \Lambda_{\beta}, \\
\operatorname{det}\left(s A_{\alpha}+(1-s) A_{\beta}\right)=s \operatorname{det} A_{\alpha}+(1-s) \operatorname{det} A_{\beta}-s(1-s) \operatorname{det}\left(A_{\alpha}-A_{\beta}\right) .
\end{gathered}
$$

This last formula for the determinant is only valid for $2 \times 2$ matrices. 
We pretend to make use of the weak continuity of det and see what conclusion we can reach. Indeed, we should have

$$
\int_{\mathbf{M}^{2 \times 2}} \operatorname{det} F d \nu(F)=\operatorname{det}\left(\int_{\mathbf{M}^{2 \times 2}} F d \nu(F)\right) .
$$

By using all the decompositions and all the formulas written above, it is elementary to arrive at

$$
\begin{gathered}
\alpha s \int_{\Lambda_{\alpha}}\left|F^{(1)}\right|^{2} d \nu_{\alpha}(F)+\beta(1-s) \int_{\Lambda_{\beta}}\left|F^{(1)}\right|^{2} d \nu_{\beta}(F) \\
=\alpha s\left|\int_{\Lambda_{\alpha}} F^{(1)} d \nu_{\alpha}(F)\right|^{2}+\beta(1-s)\left|\int_{\Lambda_{\beta}} F^{(1)} d \nu_{\beta}(F)\right|^{2}-s(1-s) \operatorname{det}\left(A_{\alpha}-A_{\beta}\right) .
\end{gathered}
$$

By Jensen's inequality, we conclude that

$$
\operatorname{det}\left(A_{\alpha}-A_{\beta}\right) \leq 0
$$

Step 2. Consider the optimization problem

subject to

$$
\text { Minimize } t\left|A_{\alpha}^{(1)}-f(x)\right|^{2}+(1-t)\left|A_{\beta}^{(1)}-f(x)\right|^{2}
$$

$$
\begin{gathered}
A=t A_{\alpha}+(1-t) A_{\beta}, \quad A_{\alpha} \in \Lambda_{\alpha}, \quad A_{\beta} \in \Lambda_{\beta}, \quad t \in[0,1] \\
\operatorname{det}\left(A_{\alpha}-A_{\beta}\right) \leq 0 .
\end{gathered}
$$

Notice that the objective functional, under these constraints, can be written as

$$
t\left|A_{\alpha}^{(1)}\right|^{2}+(1-t)\left|A_{\beta}^{(1)}\right|^{2}-2 A^{(1)} \cdot f(x)+|f(x)|^{2},
$$

so that we will be concerned about the optimization problem

$$
\text { Minimize } t\left|A_{\alpha}^{(1)}\right|^{2}+(1-t)\left|A_{\beta}^{(1)}\right|^{2}
$$

subject to

$$
\begin{gathered}
A=t A_{\alpha}+(1-t) A_{\beta}, \quad A_{\alpha} \in \Lambda_{\alpha}, \quad A_{\beta} \in \Lambda_{\beta}, \quad t \in[0,1] \\
\operatorname{det}\left(A_{\alpha}-A_{\beta}\right) \leq 0,
\end{gathered}
$$

bearing in mind that the optimal value must be corrected in the end by the term

$$
-2 A^{(1)} \cdot f(x)+|f(x)|^{2}
$$

We drop the $x$-dependence of $Q W$ in what follows.

By writing

$$
A_{\alpha}=\left(\begin{array}{c}
z \\
\alpha T z
\end{array}\right), \quad A_{\beta}=\left(\begin{array}{c}
w \\
\beta T w
\end{array}\right)
$$

for certain vectors $z, w$, it is elementary to find

$$
\begin{gathered}
z=\frac{1}{t} \frac{1}{\beta-\alpha}\left(\beta A^{(1)}+T A^{(2)}\right), \\
w=\frac{1}{1-t} \frac{1}{\alpha-\beta}\left(\alpha A^{(1)}+T A^{(2)}\right) .
\end{gathered}
$$

For simplicity, let us put

$$
A_{1}=\frac{1}{\beta-\alpha}\left(\beta A^{(1)}+T A^{(2)}\right), \quad A_{2}=\frac{1}{\alpha-\beta}\left(\alpha A^{(1)}+T A^{(2)}\right) .
$$


Then we can rewrite the above optimization problem in the form

$$
\text { Minimize }\left|A_{1}\right|^{2} \frac{1}{t}+\left|A_{2}\right|^{2} \frac{1}{1-t}
$$

subject to

$$
t \in(0,1), \quad\left|A_{1}\right|^{2} \frac{\alpha}{t^{2}}+\left|A_{2}\right|^{2} \frac{\beta}{(1-t)^{2}}-A_{1} \cdot A_{2} \frac{\alpha+\beta}{t(1-t)} \leq 0 .
$$

Notice that this last expression is precisely $\operatorname{det}\left(A_{\alpha}-A_{\beta}\right)$ and that the vectors $A_{1}$ and $A_{2}$ are constant. For future reference, put

$$
\varphi(t)=\left|A_{1}\right|^{2} \frac{\alpha}{t^{2}}+\left|A_{2}\right|^{2} \frac{\beta}{(1-t)^{2}}-A_{1} \cdot A_{2} \frac{\alpha+\beta}{t(1-t)} .
$$

Since the objective function for this new formulation is convex in $t$, it tends to $+\infty$ as $t \rightarrow 0^{+}$and $t \rightarrow 1^{-}$, and since the function determining the constraint is continuous, the minimum value sought will correspond to equality in the restriction, provided that the point of absolute minimum of the objective function on the whole interval $(0,1), t_{0}$, is such that

$$
\left|A_{1}\right|^{2} \frac{\alpha}{t_{0}^{2}}+\left|A_{2}\right|^{2} \frac{\beta}{\left(1-t_{0}\right)^{2}}-A_{1} \cdot A_{2} \frac{\alpha+\beta}{t_{0}\left(1-t_{0}\right)} \geq 0
$$

This is indeed an elementary calculus exercise. Since

$$
t_{0}=\frac{\left|A_{1}\right|}{\left|A_{1}\right|+\left|A_{2}\right|}
$$

the previous expression simplifies to

$$
(\alpha+\beta)\left(\left|A_{1}\right|+\left|A_{2}\right|\right)^{2}\left(1-\frac{A_{1}}{\left|A_{1}\right|} \cdot \frac{A_{2}}{\left|A_{2}\right|}\right) \geq 0 .
$$

Note that $A_{i}$ cannot vanish unless $A \in \Lambda_{\alpha} \cup \Lambda_{\beta}$.

Step 3. By putting together steps 1 and 2, we conclude that if $\nu$ is a gradient Young measure supported in the set where $W$ is finite and having first moment $A$, then, by decomposing $\nu$ as in step 1 and using Jensen's inequality on each part, we have

$$
\begin{aligned}
\langle W, \nu\rangle & \geq \min _{t}\left\{\left|A_{1}\right|^{2} \frac{1}{t}+\left|A_{2}\right|^{2} \frac{1}{1-t}: \varphi(t) \leq 0\right\} \\
& =\min _{t}\left\{\left|A_{1}\right|^{2} \frac{1}{t}+\left|A_{2}\right|^{2} \frac{1}{1-t}: \varphi(t)=0\right\} \\
& \geq Q W(A) .
\end{aligned}
$$

The last inequality is correct because when $\varphi(t)=0$ we obtain a first-order laminate. By taking the infimum in $\nu$ we get

$$
Q W(A)=\min _{t}\left\{\left|A_{1}\right|^{2} \frac{1}{t}+\left|A_{2}\right|^{2} \frac{1}{1-t}: \varphi(t)=0\right\} .
$$

Notice how first order laminates are the only possibility for which we can have equality.

Step 4. Computation of the previous minimum. The equation $\varphi(t)=0$ is quadratic in $t$. Indeed, it can be rewritten as

$$
\alpha(1-t)^{2}\left|A_{1}\right|^{2}+\beta t^{2}\left|A_{2}\right|^{2}-(\alpha+\beta) t(1-t) A_{1} \cdot A_{2}=0
$$


The value of this parabola for $t=0$ and $t=1$ is positive if $A$ does not belong to either $\Lambda_{\alpha}$ or $\Lambda_{\beta}$. In order for this quadratic equation to have real roots in the interval $(0,1)$, we need to demand the discriminant to be nonnegative, the leading coefficient to be (strictly) positive and the vertex to belong to $(0,1)$. After a few computations we also have

$$
\begin{aligned}
& \left(\alpha\left|A_{1}\right|^{2}+\beta\left|A_{2}\right|^{2}+(\alpha+\beta) A_{1} \cdot A_{2}\right) t^{2} \\
& \quad-\left(2 \alpha\left|A_{1}\right|^{2}+(\alpha+\beta) A_{1} \cdot A_{2}\right) t+\alpha\left|A_{1}\right|^{2}=0,
\end{aligned}
$$

or even further, bearing in mind the expressions for the vectors $A_{1}$ and $A_{2}$,

$$
\begin{aligned}
\operatorname{det} A t^{2} & -\frac{1}{\beta-\alpha}\left(\alpha \beta\left|A^{(1)}\right|^{2}-\left|A^{(2)}\right|^{2}+(\beta-\alpha) \operatorname{det} A\right) t \\
& +\frac{1}{(\beta-\alpha)^{2}}\left(\alpha \beta^{2}\left|A^{(1)}\right|^{2}+\alpha\left|A^{(2)}\right|^{2}-2 \alpha \beta \operatorname{det} A\right)=0 .
\end{aligned}
$$

Those three conditions mentioned above amount to

$$
g(A) \geq 0, \quad(\alpha+\beta) \operatorname{det} A>\left.|\alpha \beta| A^{(1)}\right|^{2}-\left|A^{(2)}\right|^{2} \mid .
$$

After some algebra, it is elementary to show that these two conditions together are equivalent to those defining the set $\Gamma$ in the statement of the theorem. For a matrix not belonging to this set, the quasiconvexification will be infinite.

Of the two roots of the above quadratic equation, by elementary continuity arguments, it is easy to show that we are interested in the one with positive root, which, after some computations, has the form given in the statement of the theorem. For the value of the minimum itself, we must calculate

$$
\left|A_{1}\right|^{2} \frac{1}{t}+\left|A_{2}\right|^{2} \frac{1}{1-t}
$$

precisely for this value of $t$. By exploiting the relationship of $t$ and $1 / t$ when $t$ is the root of a quadratic equation, and realizing that (2.1) is invariant when changing $t$ by $1-t, \alpha$ to $\beta$ and $A_{1}$ to $A_{2}$, the expression for the quasiconvexification is obtained after some careful arithmetic. Finally the term

$$
-2 A^{(1)} \cdot f(x)+|f(x)|^{2}
$$

must be added.

\section{FinAl COMMENTS}

It is worthwhile to notice that the function $Q W$ given explicitly in the theorem is, thus, a quasiconvex function, and, in particular, the set where it is finite is a quasiconvex, nonconvex set, in the sense that weak limits of gradients taking values on it will also take values within the same set. In fact, our computations amount to realizing that the polyconvexification and the rank-one convexification coincide as is typical in explicit results of this nature (see, for instance, [5], 6] among others).

It can actually be shown that the set $\Gamma$ can also be determined by using the $G$-closure of $\{\alpha \mathbf{1}, \beta \mathbf{1}\}$ (1 is the identity matrix). In fact, it is true that

$$
\Gamma=\left\{A \in \mathbf{M}^{2 \times 2}: \text { there exists } \gamma \in G[\alpha \mathbf{1}, \beta \mathbf{1}] \text { and } \gamma A^{(1)}+T A^{(2)}=0\right\} .
$$

This was noticed in [11] where the idea of introducing a potential to replace the differential constraint was indeed used and indicated. As far as we can tell, this is 
the main link between our approach and that of using relaxation based on effective conductivities of generalized layouts as in [7], [12]. Notice however that here there is no restriction on the target field $f$. Our main theorem also ensures that optimal microstructures are always rank-one laminates and that these are the only ones. This issue was also addressed in 4] and [12]. Further work may reveal a more profound connection between both perspectives.

Our computations can also be extended to cover more general cases. I plan to explore this in the near future $([2])$. On the other hand, I believe our theorem yields also the clue for the numerical approximation of optimal (or quasi-optimal) designs via relaxation of vector variational problems. I will address this issue shortly too.

\section{REFERENCES}

1. Bellido, J. C. and Pedregal, P., Optimal design via variational principles: the one-dimensional case, J. Math. Pures Appl. 80 (2001), 245-261. CMP 2001:08

2. Bellido, J. C. and Pedregal, P., in preparation.

3. Dacorogna, B., Direct methods in the Calculus of Variations, Springer, 1989. MR 90e:49001

4. Grabovsky, Y., Optimal design problems for two-phase conducting composites with weakly discontinuous objective functionals, 2001, to appear in Advan. Appl. Math.

5. Kohn, R., The relaxation of a double-well energy, Cont. Mech. Thermodyn. 3 (1991), 193-236. MR 93d:73014

6. Kohn, R. V. and Strang, G., Optimal design and relaxation of variational problems, I, II and III, CPAM 39 (1986), 113-137, 139-182 and 353-377. MR 87d:49019a MR 87d:49019b MR 87i: 49023

7. Lipton, R. and Velo, A., 2000 Optimal design of gradient fields with applications to electrostatics, in Nonlinear Partial Differential Equations and Their Applications, Collège de France Seminar, D. Cioranescu, F. Murat, and J. L. Lions, eds., Chapman and Hall/CRC Research Notes in Mathematics.

8. Murat, F., Contre-exemples pour divers problèmes où le contrôle intervient dans les coefficients, Ann. Mat. Pura ed Appl., Serie 4112 (1977), 49-68. MR 55:11123

9. Pedregal, P., Parametrized Measures and Variational Principles, Birkhäuser, Basel, 1997. MR 98e:49001

10. Pedregal, P., Optimal design and constrained quasiconvexity, SIAM J. Math. Anal. 32 (2000), 854-869. CMP 2001:08

11. Šverák, V., Lower semicontinuity of variational integrals and compensated compactness, in S. D. Chatterji, ed., Proc. ICM, vol. 2, Birkhäuser, 1994, pp. 1153-1158. MR 97h:49021

12. Tartar, L., Remarks on optimal design problems, in Calculus of Variations, Homogenization and Continuum Mechanics, G. Buttazzo, G. Bouchitté and P. Suquet, eds., World Scientific, Singapore, 1994, pp. 279-296. MR 97g:49001

13. Tartar, L., 2000 An introduction to the homogenization method in optimal design, Springer Lecture Notes in Math., vol. 1740, pp. 47-156. CMP 2001:07

Departamento de Matemáticas, ETSi Industriales, Universidad de Castilla-La ManCha, 13071 Ciudad Real, Spain

E-mail address: ppedrega@ind-cr.uclm.es 